\title{
Increased ictal perfusion of the thalamus in paroxysmal kinesigenic dyskinesia
}

\author{
S Shirane, M Sasaki, D Kogure, H Matsuda, T Hashimoto
}

\begin{abstract}
The ictal and interictal cerebral blood flow (CBF) were evaluated in a patient with right unilateral short lasting paroxysmal kinesigenic dyskinesia, by means of single photon emission computed tomography (SPECT). The patient was a 6 year old boy with no family history. During an attack, increased CBF was seen in the left thalamus. Subtraction of interictal CBF from ictal CBF disclosed a prominent increase in $\mathrm{CBF}$ in the left posterolateral part of the thalamus. This finding suggests that abnormal hyperactivity of thalamic neurons could be responsible for the pathophysiology of paroxysmal kinesigenic dyskinesia in this patient.

(F Neurol Neurosurg Psychiatry 2001;71:408-410)
\end{abstract}

Keywords: paroxysmal kinesigenic dyskinesia; SPECT; thalamus

Paroxysmal kinesigenic dyskinesia is a disease characterised by episodes of dyskinesia triggered by quick voluntary movement. The dyskinesic episodes consist of any combination of sudden dystonic posturing, choreoathetosis, and ballismus. ${ }^{1}$ Paroxysmal kinesigenic dyskinesia is associated with neither EEG abnormalities during or after attacks nor impairment of consciousness. ${ }^{2}$ The pathophysiological basis and lesion in this disease remain unclear. To obtain information on these aspects, ictal and interictal cerebral blood flow $(\mathrm{CBF})$ were studied by single photon emission computed tomography (SPECT), in a patient with paroxysmal kinesigenic dyskinesia.

\section{Case report}

The patient was a 6 year old boy. His grandfather had had Parkinson's disease from 70 years of age. The prenatal and perinatal histories were negative and early developmental milestones were normal, but he only started to utter phrases at the age of 3 years. His mother noticed that he sometimes grimaced from the age of 8 months. From the age of 1 year, he experienced paroxysmal dyskinesic attacks on the right side of his body. The attacks occurred on initiation of rapid movement, usually in the morning. During the attacks, there was no loss of consciousness. The episodes lasted less than 1 minute and occurred up to 50 times a day. The result of neurological, general physical, and laboratory examinations, and radiological studies were normal in the interictal period. He was diagnosed as having short lasting, sporadic paroxysmal kinesigenic dyskinesia, and thus was treated with carbamazepine, phenobarbital, valproate, phenytoin, or haloperidol, without improvement of the episodic dyskinesia. He was referred to our hospital for treatment at the age of 6 . He looked restless and his speech was slightly unclear. $\mathrm{He}$ had mild mental retardation. In the ictal period, he exhibited paroxysmal choreoathetotic movements involving the right side of his face and right limbs, and a dystonic posture involving the right side of the trunk and right limbs. The attacks were induced by sudden voluntary movement such as starting to speak, walk, or run. There was no loss of consciousness. The attacks occurred 10 to 30 times a day and lasted for less than 1 minute. General and neurological examinations disclosed no remarkable findings. In the interictal period, there was no laterality or abnormality of his movement, posture, or muscle tone. Neither pyramidal signs nor cerebellar signs were found. The results of laboratory examinations, cerebral MRI, and interictal EEG were normal. Ictal EEG showed no paroxysmal discharges.

Measurement of cerebral blood flow (CBF) by SPECT was performed twice (in the ictal and interictal periods). The SPECT measurements were made with a rotating gamma camera equipped with a high-resolution collimator (Siemens, MULTISPECT 3). Before the first SPECT, all medications (phenytoin and haloperidol) were tapered off. The attacks were easily provoked by sudden voluntary movement. Just after an attack started, ${ }^{123}$ I-IMP was injected. Twenty minutes later, sleep was induced with a pentobarbiturate and SPECT was carried out. After the first SPECT, he was treated again with carbamazepine. The frequency of attacks was remarkably reduced. Carbamazepine was effective. Seven months later, the second SPECT was performed; ${ }^{123}$ I-IMP was injected while he was awake and voluntarily moving both his arms. Twenty minutes later, sleep was again induced with a pentobarbiturate. No attacks occurred during the study. To allow accurate CBF subtraction, we performed MRI to match the level of the section on SPECT. Brain MRI was performed with a 3-D turbo FLASH (Siemens Magnetom Impact Expert).

\section{Results}

Ictal SPECT showed increased CBF in the left thalamus. Interictal SPECT showed increased 




Figure 1 SPECT on MRI. (A) Ictal: increased CBF in the left thalamus. (B) Interictal: increased CBF in the left medial thalamus and decreased $C B F$ in the right temporal pole. (C) Subtraction: prominent increase in CBF in the left lateral thalamus.

CBF in the left medial thalamus. Subtraction of interictal $\mathrm{CBF}$ from ictal $\mathrm{CBF}$ showed a prominent increase of $\mathrm{CBF}$ in the left posterolateral part of thalamus (fig 1).

\section{Discussion}

The pathophysiological and anatomical bases of paroxysmal kinesigenic dyskinesia remain uncertain. There is discussion about the pathophysiological basis concerning reflex epilepsy versus dysfunction of basal ganglia. Although the surface EEG recording did not show any epileptic activity, we cannot completely rule out the possibility that the dyskinesic phenomenon in this patient was epileptic. Recent studies have shown that the thalamus may play an important part in the initiation or propagation of seizures in several types of epileptic disorders. ${ }^{3-5}$ Some investigators reported that ictal SPECT showed increased perfusion in the thalamus ipsilateral to the cortical focus in patients with partial seizures. ${ }^{36}$ Our patient does not correspond to these because no cortical hyperperfusion or electroencephalographical abnormality were seen.

There have been several reports that paroxysmal kinesigenic dyskinesia is associated with specific lesions of the putamen, ${ }^{7}$ right frontotemporal region, ${ }^{8}$ globus pallidus, ${ }^{9}$ dorsal medulla oblongata, ${ }^{10}$ cervical spinal cord, ${ }^{11}$ or thalamus. ${ }^{12}{ }^{13}$ Sunohara et al reported a necropsied case in which the patient had shown exercise induced dystonia of the left limbs. ${ }^{13}$ They found necrotic lesions in the posterolateral ventral part of the right thalamus and a part of the right internal capsule. Camac et al described a patient who developed paroxysmal kinesigenic dystonic choreoathetosis after a thalamic infarct. $^{12}$ Their patient exhibited a large area of increased signal intensity in the right thalamus, including the ventral posterolateral, lateral posterior, and ventral lateral nuclei on T2 weighted MRI. Although these reports suggest that a dysfunction of the posterolateral part of the thalamus may play an important part in paroxysmal kinesigenic dyskinesia, they did not indicate whether or not there was in vivo abnormal neuronal activity in this region during the attacks. Our patient showed no remarkable lesion on MRI, but he showed a prominent increase in CBF in the left posterolateral part of the thalamus during an attack while on SPECT. From this finding, we conclude that abnormal hyperactivity of thalamic neurons contributed to the pathophysiology of paroxysmal kinesigenic 
dyskinesia in this patient. There have been a few reports of evaluation of the lesions responsible for paroxysmal kinesigenic dyskinesia by means of SPECT. Hamano et al described a 15 year old boy with a large porencephalic cyst in the left parietotemporo-occipital region who had right sided dystonic spasms induced by running. ${ }^{14} \mathrm{~A}$ cerebral blood flow study involving SPECT showed hypoperfusion of the lenticular nucleus involving the putamen and regions corresponding to the atrophy and the porencephalic cyst, but it was an interictal study only. Hayashi et al reported the results of measurement of postictal regional cerebral blood flow ( $\mathrm{rCBF}$ ) in three patients with paroxysmal kinesigenic choreoathetosis. ${ }^{15}$ Two of the three patients, who had unilateral attacks, showed a noticeable increase in $\mathrm{rCBF}$ in the basal ganglia on the contralateral side to the attacks. The difference in $\mathrm{rCBF}$ between the right and left basal ganglia in these two patients was over the mean value plus $2 \mathrm{SD}$ for seven normal subjects, and decreased with phenytoin treatment. From these results, they concluded that the neural activity of the basal ganglia is raised during the attacks in paroxysmal kinesigenic dyskinesia; but their SPECT data were for a postictal state and they did not mention which part of the basal ganglia was responsible for the attacks. Kluge et al described two patients with exercise induced paroxysmal dystonia in a pedigree showing autosomal dominant inheritance. ${ }^{16}$ Ictal and interictal cerebral perfusion were studied by means of SPECT with technetium 99m-ECD. During the motor attacks, both decreased ictal perfusion of the frontal cortex and increased cerebellar perfusion were found in these patients. In one of them, the ictal perfusion of the basal ganglia was also decreased. The hypoactive cortex areas comprised part of the frontal projection field of the mediodorsal thalamic nucleus, and they suggested that frontal hypoactivity might reflect decreased thalamic input. They concluded that frontal hypoactivity and cerebellar hyperactivity are prominent pathophysiological features of this condition. Kitagawa et al showed a decrease of ictal CBF in the basal ganglia on the contralateral side to choreoathetotic movements. ${ }^{17}$ They concluded that their findings support the hypothesis that a dysfunction of basal ganglia is relevant to the genesis of paroxysmal kinesigenic dyskinesia. Our findings also support this hypothesis, although in our patient ictal SPECT disclosed an increase of cerebral blood flow in the thalamus. Some investigators reported changes in CBF on SPECT during voluntary movement in normal subjects. ${ }^{18-20}$ Their subjects showed a contralateral increase in tracer uptake in the insular cortex, primary motor area, supplementary motor area, medial primary sensorimotor area, striatum, cerebellar vermis, and visual cortex. Our patient had not moved his upper limbs voluntarily during the loading of ${ }^{123}$ I-IMP in the examination. From these facts, we conclude that voluntary movement is not responsible for our finding. Recent methodological advances in neuroscience have provided substantial new information on the neural circuit of the basal ganglia. ${ }^{21-23}$ Chorea and ballism appear as a consequence of functional inactivation or a lesion of the subthalamic nucleus leading to reduced neuronal activity in both the globus pallidus pars externa and pars interna. ${ }^{24}$ Dystonia is viewed as a consequence of the release of premotor cortical activity from thalamic control, which is supported by recent positron emission tomographic studies. ${ }^{25}$ In the present case, as judged from the results of SPECT, neuronal activity in the posteriolateral part of the left thalamus showed primary or secondary increases during the attacks. If the inhibition of the globus pallidus pars interna/ substantia nigra pars reticulata is insufficient, when these thalamic neurons activate the cortical motor area in the frontal lobes, paroxysmal dyskinesia could occur.

We are very grateful to Dr Masaya Segawa for his important suggestion.

1 Demirkiran M, Jankovic J. Paroxysmal dyskinesias: clinical features and classification. Ann Neurol 1995;38:571-9.

2 Lance JW. Familial paroxysmal dystonic choreoathetosis and its differentiation from related syndromes. Ann Neurol 1977;2:285-93.

3 Marks DA, Katz A, Hoffer P, et al. Localization of extratemporal epileptic foci during ictal single photon emission computed tomography. Ann Neurol 1992;31:250-5.

4 Chugani HT, Rintahaka PJ, Shewmon DA. Ictal patterns of cerebral glucose utilization in children with epilepsy. Epilepsia 1994;35:813-22.

5 Vonck K, Boon P, Laere KV, et al. Acute single photon emission computed tomographic study of vagus nerve stimulation in refractory epilepsy. Epilepsia 2000;41:601-9.

6 Yune MJ, Lee JD, Ryu YH, et al. Ipsilateral thalamic hypoperfusion on interictal SPECT in temporal lobe epilepsy. $\mathcal{F}$ Nucl Med 1998;39:281-5.

7 Merchut MP, Brumlik J. Painful tonic spasms caused by putaminal infarction. Stroke 1986;17:1319-21.

8 Gilroy J. Abnormal computed tomograms in paroxysmal kinesigenic choreoathetosis. Ann Neurol 1982;39:779-80.

9 Micheli F, Pardal MMF, Parera IC, et al. Sporadic paroxysmal dystonic choreoathetosis associated with basal ganglia mal dystonic choreoathetosis associated

10 Riley DE. Paroxysmal kinesigenic dystonia associated with a medullary lesion. Mov Disord 1996;11:738-40.

11 Cosentino C, Torres L, Flores M, et al. Paroxysmal kinesigenic dystonia and spinal cord lesion. Mov Disord 1996;11:453-5.

12 Camac A, Greene P, Khandji A. Paroxysmal kinesigenic dystonic choreoathetosis associated with a thalamic infarct. Mov Disord 1990;5:235-8.

13 Sunohara N, Mukoyama M, Mano Y, et al. Action-induced thythmic dystonia: an autopsy case. Neurology 1984;34: $321-7$.

14 Hamano S, Tanaka Y, Nara T, et al. Paroxysmal kinesigenic choreoathetosis with prenatal brain damage. Acta Pediatr faponica 1995;37:401-4.

15 Hayashi R, Hanyu N, Yahikozawa H, et al. Ictal muscle discharge pattern and SPECT in paroxysmal kinesigenic 89-94.

16 Kluge A, Kettner B, Zschenderlein R, et al. Changes in perfusion pattern using ECD-SPECT indicate frontal lobe and cerebellar involvement in exercise-induced paroxysmal dystonia. Mov Disord 1998;13:125-34

17 Kitagawa N, et al. Ictal 99mTC-HMPAO-SPECT in a case of paroxysmal kinesigenic choreoathetosis. Rinsho Shinkeigaku 1998;38:767-70

18 Ebmeier KP, Murray CL, Dougall NJ, et al. Unilateral voluntary hand movement and regional cerebral uptake of technetium-99m-exametazione in human control subjects. f Nucl Med 1992;33:1623-7.

19 Fukuyama H, Ouchi Y, Matsuzaki S, et al. Brain functional activity during gait in normal subjects: a SPECT study. activity during gait in norma
Neurosci Lett 1997;228:183-6.

20 Williamson JW, Nobrega AC, McColl R, et al. Activation of the insular cortex during dynamic exercise in humans. $\mathcal{f}$ Physiol 1997;503:277-83.

21 Obeso JA, Rodriguez MC, DeLong MR. The basal ganglia and new surgical approaches for Parkinson's disease. $A d v$ Neurol 1997;74:3-18.

22 Nakano K, Kayahara T, Ushiro H, et al. Some aspects of basal ganglia-thalamocortical circuitry and descending outputs of the basal ganglia. Monographs in Neural Sciences 1995;14:134-46.

23 Alexander GE, Crutcher MD. Functional architecture of basal ganglia circuits: neural substrates of parallel processing. TINS 1990;7:266-71.

24 Crossman AR, Sambrook MA, Jackson A. Experimental hemichorea/hemiballismus in the monkey: studies on the intracerebral site of action in a drug-induced dyskinesia. Brain 1984;107:579-96.

25 Ceballos-Baumann AO, Passingham RE, Marsden CD, et al. Motor reorganization in acquired hemidystonia. Ann Neurol 1995;37:746-57. 